\title{
EVALUAR PARA APRENDER: ESTADO ACTUAL DE CATORCE ASIGNATURAS EN LA UNIVERSIDAD ESTATAL A DISTANCIA DE COSTA RICA
}

\section{ASSESSMENT FOR LEARNING: CURRENT STATUS OF FOURTEEN COURSES AT UNIVERSIDAD ESTATAL A DISTANCIA OF COSTA RICA}

\section{Ana Cristina Umaña-Mata ${ }^{1}$, acumanam@uned.ac.cr Xinia Calvo-Cruz ${ }^{2}$,xcalvo@uned.ac.cr Natalia Salas-Quirós ${ }^{3}$, nsalas@uned.ac.cr Universidad Estatal a Distancia, Costa Rica}

\author{
Volumen 8, Número 2 \\ 30 de noviembre de 2017 \\ pp. $24-61$
}

Recibido: 6 de setiembre de 2017

Aprobado: 04 de octubre de 2017

\footnotetext{
${ }^{1}$ Dra. Ana Cristina Umaña Mata, Doctora en Educación, Magister en Planificación Curricular, Coordinadora del Programa de Apoyo Curricular y Evaluación de los Aprendizajes. Universidad Estatal a Distancia, Costa Rica. Correo electrónico: acumanam@uned.ac.cr

${ }^{2}$ Mag. Xinia Calvo Cruz, Magister en Planificación Curricular, Asesora Curricular, Trabaja en el Programa de Apoyo Curricular y Evaluación de los Aprendizajes. Correo electrónico: xcalvo@uned.ac.cr

${ }^{3}$ Mag. Natalia Salas Quirós, Magister en Planificación Curricular, Asesora Curricular, Trabaja en el Programa de Apoyo Curricular y Evaluación de los Aprendizajes. Correo electrónico: nsalas@uned.ac.cr
}

Evaluar para Aprender: estado actual de catorce asignaturas en la Universidad Estatal a

Distancia de Costa Rica

Ana Cristina Umaña-Mata, Xinia Calvo-Cruz,

Natalia Salas-Quirós

DOI : http://dx.doi.org/10.22458/caes.v8i2.1809

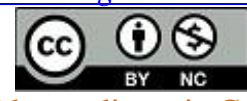




\section{Resumen}

La Universidad Estatal a Distancia de Costa Rica (UNED) a partir del 2012, en su plan de desarrollo académico presta especial atención al tema de evaluación de los aprendizajes, desde los principios del evaluar para aprender. Como parte de una investigación, se realizó un diagnóstico del estado de 14 asignaturas que fueron objeto de estudio. En este diagnóstico se consideró la presencia de los criterios mínimos establecidos por el Programa de Apoyo Curricular y Evaluación de los Aprendizajes (PACE), que se deben tomar en cuenta, cuando se asume la propuesta teórica de evaluar para aprender. El interés de este documento es presentar los resultados generales de esta etapa.

El trabajo se enmarca dentro del modelo propio de la investigación acción, la cual, según Suárez (2002), tiene como propósito la mejora de la práctica. Esa mejora se enmarca, además de la práctica, en los contextos en que se realiza. En consecuencia, "la pretensión de mejorar acciones, ideas y contextos; son un marco idóneo como punto de unión entre la teoría y la práctica, la acción y la reflexión" (citado por Campos y Madriz, 2015, p 25).

Los hallazgos del diagnóstico evidencian el desconocimiento de las personas participantes en la investigación (equipo de coinvestigación), ya que estas ignoran el marco teórico- metodológico de evaluar para aprender, lo que llevó en algunos casos a que se presentaran dificultades para la comprensión de las implicaciones de cada criterio, relacionado con las características y funciones del evaluar para aprender, lo que a su vez acarreó dificultades para identificar las insatisfacciones en las asignaturas de cada equipo.

Palabras clave: evaluar para aprender; investigación acción; educación a distancia; evaluación formativa; evaluación formadora; aprender a aprender.

\section{Abstract}

The Universidad Estatal a Distancia of Costa Rica (UNED), since 2013 in its academic development plan pays special attention to the evaluation of learning processes, oriented from the perspective of assessment for learning. For this reason, the university worked on a diagnosis as the first stage in the action - research model that is taking place in Programa de Apoyo Curricular y Evaluación de los Aprendizajes (PACE) and in the different schools of the university. For this, there was a diagnosis of the state of each of the fourteen courses that participated in this first stage, about the minimum criteria that has to be taken into consideration when there is an assumption of the theoretical proposal of assessment for learning. The interest of this paper, is to present the general results of this diagnosis.

Evaluar para Aprender: estado actual de catorce asignaturas en la Universidad Estatal a

Distancia de Costa Rica

Ana Cristina Umaña-Mata, Xinia Calvo-Cruz,

Natalia Salas-Quirós

DOI : http://dx.doi.org/10.22458/caes.v8i2.1809

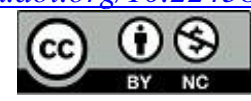

Artículo protegido por licencia Creative Commons 
This investigation is framed into the action - research model, which aims to improve the practice, Suárez (2002). This improvement is framed in addition to the practice in the contexts in which it is performed. Consequently, "the pretension to improve actions, ideas and contexts, are an ideal framework as a point of union between theory and practice, action and reflection" (quoted by Campos y Madriz, 2015, p 25).

The findings of the diagnosis, show the need for a theoretical-methodological framework to evaluate for learning for the university that would permit to guide the institutional evaluation processes.

From the information gathered in this diagnosis, it can be said that the people involved in the research (co-investigators) are unaware of the theoretical-methodological framework of evaluate for learning, which led in some cases to difficulties in understanding the implications of each criterion related to the characteristics and functions of the evaluate for learning, which in turn into difficulties to identify dissatisfactions in the subjects of each team.

Keywords: assessment for learning; action research; distance education; formative evaluation; formative evaluation; learning to learn

\section{Introducción}

La evaluación de los aprendizajes en la educación superior es una temática a la cual se le ha prestado especial atención en los últimos años, debido a la necesidad de las instituciones educativas en dar respuesta a las tendencias de la sociedad actual, que exige la formación de profesionales integrales, capaces de aprender a aprender permanentemente.

En relación con el tema, la Universidad Estatal a Distancia de Costa Rica (UNED) lleva a cabo a partir del 2012 una propuesta para la evaluación de los aprendizajes, orientada desde los principios del evaluar para aprender. En el PACE, se inicia el proceso de investigación-acción, cuyo propósito fue establecer las posibilidades de implementación de este marco teórico-metodológico en el modelo educativo de dicha institución. Para los

Evaluar para Aprender: estado actual de catorce asignaturas en la Universidad Estatal a

Distancia de Costa Rica

Ana Cristina Umaña-Mata, Xinia Calvo-Cruz,

Natalia Salas-Quirós

DOI : http://dx.doi.org/10.22458/caes.v8i2.1809

\section{(c) $(1)$ (8)}


efectos del caso, fue necesario en primera instancia llevar a cabo un diagnóstico del estado de cada una de las asignaturas seleccionadas con respecto a los criterios mínimos que se deben considerar cuando se asume la propuesta teórica de evaluar para aprender. Por tanto, el interés de este documento es presentar los resultados generales del diagnóstico.

\section{Referente contextual}

Desde sus inicios, la UNED ha mantenido un interés por el análisis y mejora de sus prácticas educativas, en especial del proceso de evaluación de aprendizajes. Es así, que para el año de 1978 la Vicerrectoría Académica crea la Política y Sistema de Evaluación, en la cual concibe la evaluación de aprendizaje, como un proceso sistemático y constante, al que llamaron un sistema de evaluación sumativo y formativo, orientado hacia el uso de pruebas escritas, tareas y autoevaluaciones, así como formularios para "autoobservación" y "de dificultades", éstos últimos referidos a la función formativa de la evaluación.

Cuatro años más tarde, se lleva a cabo el I seminario sobre evaluación de los aprendizajes (1982), donde se conceptualiza la evaluación sumativa como el "proceso sistemático de recolección de información al final de una unidad, curso o semestre, para establecer en qué medida se logran los conocimientos y habilidades enunciadas en los objetivos propuestos" (p.3). Mientras que la evaluación formativa fue entendida como aquella en la que "cada estudiante evalúa el grado de avance en el logro de los objetivos propuestos para la toma de decisiones adecuadas durante el desarrollo del proceso de enseñanzaaprendizaje, que le permitan reorientar y superar las dificultades específicas de aprendizaje" (p.3).

Evaluar para Aprender: estado actual de catorce asignaturas en la Universidad Estatal a

Distancia de Costa Rica

Ana Cristina Umaña-Mata, Xinia Calvo-Cruz,

Natalia Salas-Quirós

DOI : http://dx.doi.org/10.22458/caes.v8i2.1809

\section{(c) $($ ) $(8)$}


Con esta conceptualización, se incorporan como actividades evaluativas los proyectos y las tareas de ejecución, así como los solucionarios de pruebas, tareas y los ejercicios de autoevaluación, como una opción para dar respuesta a un proceso donde se empieza a dar al estudiantado un protagonismo en su aprendizaje. Sin embargo, continúan los esfuerzos centrados en la medición y el uso de pruebas escritas.

Posteriormente, y hasta el año 2000, la universidad retoma la temática con la aprobación de la moción 025 del II congreso universitario, de la que se destaca la necesidad de creación de un modelo pedagógico que replantee el sistema de evaluación vigente hasta ese momento; que toma en cuenta la consideración del aprendizaje significativo, el establecimiento de programas de capacitación y actualización permanente del personal docente, el establecimiento de modelos de evaluación e instrumentos de medición, así como la realización de diversas acciones para asegurar la calidad de estos instrumentos, su aplicación y el uso de la información derivada para la toma de decisiones.

Es así, como, para el año 2004 se crea el modelo pedagógico de la UNED, en el que se incorporan los conceptos de regulación y autorregulación, basados en principios de autoaprendizaje, construcción conjunta y colaboradora de los aprendizajes, teniendo como meta el desarrollo de la competencia de aprender a aprender. Esto orienta el papel de la docencia hacia la facilitación y mediación pedagógica, pero retomando la división de la evaluación en sumativa y formativa.

Un año después se crea el Reglamento de Gestión Académica (2005), que establece que "el diseño [curricular] de una carrera o curso [asignatura] debe contemplar, [...] la autorregulación de los aprendizajes" (artículo 14), de igual modo establece la

Evaluar para Aprender: estado actual de catorce asignaturas en la Universidad Estatal a

Distancia de Costa Rica

Ana Cristina Umaña-Mata, Xinia Calvo-Cruz,

Natalia Salas-Quirós

DOI : http://dx.doi.org/10.22458/caes.v8i2.1809

\section{(c) $(1)(2)$}


responsabilidad de la persona encargada de cátedra y de carrera de plantear el tipo de evaluación, y velar porque se permita el logro de los rasgos del perfil académicoprofesional (artículos 29 y 30 inciso b). Aunado a esto, da la función de asesoría en esta materia al Programa de Apoyo Curricular y Evaluación de los Aprendizajes (PACE) (artículos 32 y 40).

Para el año 2012, se crea el Reglamento General Estudiantil, el cual posee un capítulo exclusivo para normar el proceso de evaluación de los aprendizajes. En este se define la evaluación como "proceso sistemático de obtención de información válida y útil para formular juicios valorativos acerca de los efectos del proceso de formación en cada estudiante" citar la página. Adicionalmente, establece el carácter continuo, formativo y sumativo de la evaluación, aunque en la mayor parte de los artículos que componen este reglamento se centran en el carácter sumativo de las pruebas escritas, por tanto, se puede ver la prevalencia del modelo evaluativo con el cual nació la institución.

De igual manera, en el 2012, la Vicerrectoría Académica presenta el Plan de Desarrollo Académico (2012-2017), el cual enfoca la evaluación de los aprendizajes, desde el interés por el aprendizaje. En este sentido, en la UNED (2013), se concibe la evaluación para aprender como un proceso continuo, formativo y formador, inmerso en el aprendizaje que se fundamenta en el aprender a aprender; el cual es, a su vez, un elemento orientador del diseño curricular de asignaturas, del diseño de materiales, de las acciones de mediación pedagógica, así como de la formación de una persona autónoma y capaz de seguir aprendiendo por sí misma.

Evaluar para Aprender: estado actual de catorce asignaturas en la Universidad Estatal a Distancia de Costa Rica Ana Cristina Umaña-Mata, Xinia Calvo-Cruz,

Natalia Salas-Quirós

DOI : http://dx.doi.org/10.22458/caes.v8i2.1809

\section{(c) $(1)(8)$}


Así, se presentan como características del evaluar para aprender las siguientes: auténtica, alternativa, integral, continua, adecuada al contexto, diagnóstica, formativa y sumativa, que involucra autoevaluación, coevaluación y heteroevaluación, centrada en el estudiante, sistemática, que considere los roles profesionales, ritmos y estilos de aprendizaje, válida y confiable (UNED, 2013).

No obstante, fue en el año 2015 que se presentan los Lineamientos de Política Institucional 2015-2019. De esto, se plantea como tendencia a la fecha la concepción tradicional de la evaluación centrada en la certificación de aprendizajes, y plantea en los lineamientos 68 y 69, que la evaluación debe atender las características disciplinarias y de aprendizaje de cada asignatura, promover el pensamiento crítico, el uso de actividades evaluativas alternativas, la innovación en la forma de administrar las evaluaciones, así como la mejora de los instrumentos de evaluación, de éstos últimos señalan puntualmente la elaboración de pruebas escritas.

Desde este marco, y específicamente, para coadyuvar al cumplimiento de la política institucional en la innovación educativa, relacionada con el evaluar para aprender (Plan de Desarrollo Académico, 2012, p. 57), en la que se plantea, generar líneas y procesos de investigación, considerando "nuevas posturas teóricas, diversidad de población universitaria, atención a las necesidades educativas, entornos virtuales de aprendizaje, pruebas psicométricas y rendimiento académico, entre otros" (UNED, 2012, p. 22), es que el PACE se propuso en el año 2015 llevar a cabo un proyecto de investigación que permita establecer aquellas limitaciones y posibilidades de implementar el marco teóricometodológico de evaluar para aprender, según lo establecido en el Plan de Desarrollo

Evaluar para Aprender: estado actual de catorce asignaturas en la Universidad Estatal a

Distancia de Costa Rica

Ana Cristina Umaña-Mata, Xinia Calvo-Cruz,

Natalia Salas-Quirós

DOI : http://dx.doi.org/10.22458/caes.v8i2.1809

\section{(c) $($ ) $(8)$}


Académico, que servirán de insumo y referente a la Vicerrectoría Académica, antes de plantear los respectivos lineamientos sobre el "Evaluar para Aprender".

Con esto, se busca guiar la actuación de las instancias académicas de la universidad, definir los aspectos técnicos, operativos, así como las pautas, con el fin de llevar a cabo la evaluación para aprender, que estén basadas en los resultados obtenidos, como producto de la implementación en las asignaturas de un análisis correspondiente con su marco teórico-metodológico.

Esto se lleva a cabo en respuesta a la función dada al PACE, quien es la instancia de la Vicerrectoría Académica encargada de orientar y asesorar a dicha vicerrectoría y a las escuelas, en lo referente a cambios y mejoras en la evaluación de los aprendizajes (Reglamento de Gestión Académica artículo 32 y artículo 40, inciso 4).

\section{Referentes conceptuales}

\subsection{Principios epistemológicos de la evaluación para aprender en la UNED.}

El modelo pedagógico de la UNED (2004) tiene como centro el estudiantado, quien se considera protagonista de su proceso de aprendizaje. Bajo esta premisa, se tiene como principios epistemológicos los siguientes:

- El conocimiento se origina de la interacción dialéctica entre el sujeto cognoscente y el objeto, dentro de un marco histórico contextual del que forma parte el sujeto.

- El sujeto aprendiz es un ente activo que interpreta y reinterpreta la realidad de forma continua y dinámica.

- Es un ser en contexto (UNED, 2004, p. 25).

Evaluar para Aprender: estado actual de catorce asignaturas en la Universidad Estatal a

Distancia de Costa Rica

Ana Cristina Umaña-Mata, Xinia Calvo-Cruz,

Natalia Salas-Quirós

DOI : http://dx.doi.org/10.22458/caes.v8i2.1809

\section{(c) $\underset{\mathrm{BY}}{\mathrm{Br}}(\mathrm{SC}$}


Con este referente, se puede decir que toda persona tiene una serie de estructuras cognitivas construidas a partir de sus conocimientos previos, las cuales se modifican y se transforman a partir de la interacción que establece con el objeto de conocimiento, lo que da como resultado, la asimilación de los nuevos conocimientos, la interpretación y reinterpretación de la realidad, desde aprendizajes significativos, funcionales y transferibles. Dicho proceso de construcción de conocimientos estará a su vez influenciado por el contexto en el que se encuentra inmersa la persona estudiante, donde el lenguaje, las herramientas culturales y la interacción juegan un papel fundamental para el proceso de aprendizaje (Chadwick, 2001). Asimismo, se ha de tener en cuenta que en esta construcción de conocimiento intervienen no sólo aspectos cognitivos, sino también motivacionales, afectivos y sociales.

Desde esta perspectiva, el proceso de aprender va a estar dado desde lo intrapsicológico e interpsicológico para la internalización de los conocimientos, desde el cual se han de plantear una serie de estrategias didácticas que permitan a cada estudiante alcanzar la zona de desarrollo óptimo, para lo que adquieren relevancia los procesos de regulación y autorregulación del aprendizaje. Así, esta forma de entender el proceso de construcción de conocimientos conlleva una mirada distinta acerca de la evaluación, que debe estar orientada hacia el favorecimiento del aprendizaje más que a la sola certificación o la calificación. Esto es lo que se ha llamado evaluar para aprender.

\subsection{Características y funciones de evaluar para aprender}

Broadfoot, citado en Wiliam (2011), define la evaluación para aprender como "el proceso de búsqueda e interpretación de evidencias para ser usada por los aprendices y sus

Evaluar para Aprender: estado actual de catorce asignaturas en la Universidad Estatal a

Distancia de Costa Rica

Ana Cristina Umaña-Mata, Xinia Calvo-Cruz,

Natalia Salas-Quirós

DOI : http://dx.doi.org/10.22458/caes.v8i2.1809

\section{(c) $($ ) $(8)$}


profesores para decidir donde se encuentran los aprendices en su aprendizaje, donde necesitan ir y cuál es la mejor manera de llegar alli" (p. 10)

Adicionalmente, en la conferencia internacional de evaluación para el aprendizaje en Dunedin (2009) se acordó la siguiente definición: "Evaluar para aprender es una parte de la práctica diaria de los estudiantes, profesores y pares que busca, reflexiona y responde a información desde el diálogo, la demostración y la observación de manera que mejore el aprendizaje continuo" (Wiliam, 2011, p. 10).

Desde este punto de vista, la evaluación puede considerarse, entonces, como una de las actividades intrínsecas al proceso de aprendizaje, la cual tiene como finalidad la promoción de procesos de reflexión, derivados de la información recopilada sobre qué se ha aprendido, qué se requiere aprender y cómo se puede mejorar, a fin de replantear o hacer los ajustes necesarios en los procesos de enseñanza y de aprendizaje. En ella es necesaria la participación de los diferentes actores del proceso pedagógico (profesorado, estudiantado y sus pares), y de ese modo tener una visión más amplia de dicho proceso, en el que se logre el desarrollo de las capacidades cognitivas y emocionales requeridas para aprender a aprender, así como las mejoras a las diversas estrategias didácticas utilizadas.

Al respecto Álvarez menciona (en Sacristán, 2010, p.359)

[...] la evaluación que se deriva de estos principios implica el fomento del debate sobre ideas nuevas y del pensamiento divergente, creativo, pensamiento autónomo, crítico (análisis, elaboración de hipótesis, contraste de fuentes de

Evaluar para Aprender: estado actual de catorce asignaturas en la Universidad Estatal a Distancia de Costa Rica Ana Cristina Umaña-Mata, Xinia Calvo-Cruz,

Natalia Salas-Quirós

DOI : http://dx.doi.org/10.22458/caes.v8i2.1809

\section{(c) $\underset{\mathrm{BY}}{\mathrm{BC}}(\mathrm{NC}$}


información, distanciamiento de la inmediatez de las fuentes), con posibilidades de establecer relaciones variadas y soluciones diferentes.

Sobre este particular, la evaluación para aprender desde el Plan de Desarrollo Académico 2012-2017 se caracteriza por ser integral e integrada (UNED, 2013, p. 40). Integral en la medida que permite relacionar la teoría con la práctica, así como lo cognitivo con lo afectivo, es decir, propiciar la reflexión acerca del propio proceso de aprendizaje. E integrada porque es parte del proceso de aprendizaje, y, por tanto, se realiza de forma continua, dejando de ser solamente un evento al final de un período académico, por ejemplo.

Adicional a lo anterior, la evaluación desde el Plan de Desarrollo Académico, se caracteriza por ser auténtica y alternativa; es decir, incorpora diversas actividades evaluativas que toman en cuenta el contexto socio-profesional, utilizando para ello simulaciones o prácticas in situ. Esto implica el uso de diversas herramientas, lenguajes y metodologías propias del campo profesional, el trabajo colaborativo e individual, así como el uso de procesos de autoevaluación, coevaluación y heteroevaluación.

En este sentido, la evaluación para aprender posee dos funciones sustanciales: una formativa y una formadora, que, en su característica de integral, deben estar presentes de manera amalgamada en el proceso de aprendizaje. Estas dos funciones se describen a continuación.

Evaluar para Aprender: estado actual de catorce asignaturas en la Universidad Estatal a Distancia de Costa Rica Ana Cristina Umaña-Mata, Xinia Calvo-Cruz,

Natalia Salas-Quirós

DOI : http://dx.doi.org/10.22458/caes.v8i2.1809

\section{(c) $(1)(8)$}




\subsubsection{La función formadora de la evaluación para el aprendizaje.}

La función formadora de la evaluación tiene que ver con los procesos de autorregulación del aprendizaje, cuya finalidad es el desarrollo de la capacidad de aprender a aprender a lo largo de la vida. Dichos procesos de autorregulación corresponden a "un conjunto de procesos específicos de planificación, monitorización y evaluación de la propia actuación propios de tareas de aprendizaje específicas y prototípicas [...]" (Monereo y Badia, 2013, p.23)

Al respecto, Sanmartí y Jorba en Pozo y Monereo (2000) afirman que desde esta función formadora, la evaluación "pretende formar al alumnado en sus procesos de pensamiento $y$ de aprendizaje ayudándole a construir su propio sistema personal de aprendizaje" ( $p$. 303), para ello es necesario propiciar en el estudiantado el uso de estrategias de metacognición, mediante las orientaciones que se brindan en la realización de las actividades de aprendizaje que se le proponen, de manera que pueda identificar con claridad cuál es la finalidad de la actividad, cómo debe llevarse, qué conocimientos se requieren y con qué debe realizarla, a fin de que el estudiantado tome decisiones y establezca sus estrategias de aprendizaje.

Sobre este particular, Colmenares (2012) afirma que la evaluación formadora

[...] ofrece indicadores al alumno para que interiorice criterios de auto-evaluación y auto-regulación de su propio aprendizaje, desde esta perspectiva la evaluación debe ser favorecedora de la autonomía del aprendizaje del alumno, es decir, que potencie el autorreflexión sobre sus propios procesos de construcción de aprendizaje. (p.127)

Evaluar para Aprender: estado actual de catorce asignaturas en la Universidad Estatal a

Distancia de Costa Rica

Ana Cristina Umaña-Mata, Xinia Calvo-Cruz,

Natalia Salas-Quirós

DOI : http://dx.doi.org/10.22458/caes.v8i2.1809

\section{(c) $($ ) $(8)$}


No obstante, este proceso de autorregulación, desde la teoría de la actividad y del socioconstructivismo, se verá favorecido en la medida en que se esté en interacción con los demás, por tal motivo, la función formativa de la evaluación no puede estar separada de la función formadora.

\subsubsection{La función formativa de la evaluación para el aprendizaje.}

Autores como Álvarez en Sacristán (2010) y Bordas y Cabrera (2001) coinciden en que la evaluación adquiere un carácter formativo al estar en función de quien aprende, para lo cual es condición esencial la participación activa y compromiso del estudiantado en todo el proceso evaluativo. En este sentido, Popham (2013) afirma la evaluación formativa es un proceso planificado en el que tanto los profesores como los alumnos usan la evidencia basada en la evaluación para realizar ajustes en lo que están haciendo (p.14).

Álvarez en Sacristán (2010) señala como condiciones de la evaluación formativa las siguientes:

- Que sea justa y ecuánime

- Que sea recurso de mejora, de motivación.

- Que la información sea útil y a tiempo para que cada alumno comprenda sus debilidades, sus errores, sus deficiencias.

- Que preste atención a cómo el alumno aprende, no sólo lo que el alumno aprende, y preste atención a las estrategias de éxito que utiliza el alumno para obtener un buen resultado.

- Que los criterios en los que se basa para la valoración y la calificación sean explícitos, claros, inteligibles, transparentes.

Evaluar para Aprender: estado actual de catorce asignaturas en la Universidad Estatal a

Distancia de Costa Rica

Ana Cristina Umaña-Mata, Xinia Calvo-Cruz,

Natalia Salas-Quirós

DOI : http://dx.doi.org/10.22458/caes.v8i2.1809

\section{(c) ${ }_{\mathrm{BY}}($ SC}


- Que permita al profesorado ajustar con más acierto su enseñanza a las necesidades y dificultades que el alumno encuentra en su aprendizaje y pueda introducir cambios de mejora en el proceso.

- Que la evaluación esté integrada en el proceso y en las actividades normales de enseñanza y de aprendizaje, y contribuya a mejorarlos.

- Que actúe en contextos de confianza, de respeto y de responsabilidad compartida.

- Que informe y prepare a quien aprende sobre la capacidad de aprendizajes futuros, no sólo sobre aprendizajes pasados.

- Que estimule el aprendizaje y consiga que el alumno centre su atención y sus esfuerzos en el valor de los contenidos que aprende, no tanto en la calificación.

- Que implique a todos, profesores y alumnos, en la elaboración de las preguntas o de los problemas, en la implementación y en el éxito de los resultados (pp.365-368).

En la siguiente tabla 0 de Bordas y Cabrera (2001) se resumen las principales características de ambas funciones de la evaluación.

Tabla 0. Diferencias entre evaluación formativa y evaluación formadora.

\section{EVALUACION FORMATIVA}

- Intervención docente

- Iniciativa del docente

- Surge del proceso de enseñanza

- Proviene de fuera

- Repercute en el cambio positivo desde "fuera"

\section{EVALUACIÓN FORMADORA}

- Parte del propio discente y/o orientada por el docente

- Iniciativa del discente

- Surge de la reflexión del discente

- Proviene de dentro

- Repercute en el cambio positivo desde "dentro"

Fuente: Bordas y Cabrera (2001, p. 33)

Evaluar para Aprender: estado actual de catorce asignaturas en la Universidad Estatal a

Distancia de Costa Rica

Ana Cristina Umaña-Mata, Xinia Calvo-Cruz,

Natalia Salas-Quirós

DOI : http://dx.doi.org/10.22458/caes.v8i2.1809

\section{(c) $(1)(8)$}




\section{Metodología del diagnóstico}

La investigación se enmarca dentro del modelo propio de la investigación acción, la cual, según Suárez (2002) tiene como propósito la mejora de la práctica. Esa mejora se enmarca en los contextos en que se realiza. Además, Suárez agrega: "la pretensión de mejorar acciones, ideas y contextos; son un marco idóneo como punto de unión entre la teoría y la práctica, la acción y la reflexión" (citado por Campos y Madriz, 2015, p.25).

La investigación acción tiene varias modalidades; entre ellas se cita la modalidad técnica. Esta modalidad, es la seleccionada para el desarrollo del proyecto de investigación del PACE. Según Murillo (2011), este tipo de investigación se caracteriza por la generación de prácticas sociales mucho más eficaces mediante el involucramiento de grupos interesados en realizar cambios y que, a su vez, estén en disposición de trabajar orientados por el modelo que va a ser diseñado por el grupo de expertos. Por tanto, es necesario que tanto los propósitos, los objetivos, así como la ruta metodológica a seguir, se encuentre previamente elaborada por parte del equipo experto.

Al respecto, Colás y Buendía (1998, p.292) indican que la modalidad técnica de la investigación-acción tiene como objetivo:

[...] hacer más eficaz la práctica educativa, posibilitando y animando a que los responsables de cualquier actividad participen y colaboren con un agente externo a fin de mejorar sus acciones [...] el programa de trabajo es diseñado por expertos, el cual establece los objetivos del mismo y el desarrollo metodológico que se ha de seguir

Evaluar para Aprender: estado actual de catorce asignaturas en la Universidad Estatal a

Distancia de Costa Rica

Ana Cristina Umaña-Mata, Xinia Calvo-Cruz,

Natalia Salas-Quirós

DOI : http://dx.doi.org/10.22458/caes.v8i2.1809

\section{(c) ${ }_{\mathrm{BY}}(2)$}


En ese sentido, el equipo de investigación del PACE, se convierte en "el agente externo" a la situación en estudio. Por su experticia en la temática de evaluación de los aprendizajes es quien diseña el programa de trabajo que se ha de seguir, específicamente, con la atención de la temática del evaluar para aprender. A este equipo de participantes, en adelante se llamará equipo de investigación principal y a los equipos participantes equipo de coinvestigación.

El proyecto integra varios equipos de coinvestigación responsables de ejecutar el programa de trabajo diseñado por el equipo de especialistas del PACE. Sin embargo, cada grupo ejecuta su propia investigación, por lo que se ubican en el nivel de participantes, cuyo fin es que, en su primer ciclo, apliquen el marco teórico-metodológico del evaluar para aprender en cada una de las asignaturas objeto de estudio. Para efectos de este artículo, se expone el trabajo realizado en la primera etapa llamada: diagnóstico.

\subsection{Selección de los equipos coinvestigadores}

Para la selección de los participantes de los equipos coinvestigadores, el equipo principal realizó el siguiente procedimiento:

a) Se convocó a una reunión a las personas, que en las diferentes escuelas de la UNED tienen a su cargo el seguimiento de los procesos de evaluación de los aprendizajes. En este espacio, se les explicó en detalle el objetivo de la investigación, y se solicitó discutirlo con las personas directoras de las escuelas para definir el mecanismo de selección de los posibles equipos coinvestigadores que podrían participar en el proyecto en representación de cada una de las escuelas.

Evaluar para Aprender: estado actual de catorce asignaturas en la Universidad Estatal a Distancia de Costa Rica Ana Cristina Umaña-Mata, Xinia Calvo-Cruz,

Natalia Salas-Quirós

DOI : http://dx.doi.org/10.22458/caes.v8i2.1809

\section{(c) $(1)(8)$}


b) Los equipos de personas seleccionados por escuela fueron convocados a una sesión informativa sobre el proyecto, con el fin de que cada uno decidiera si existía interés de participar en éste.

c) Se definieron criterios de selección para las personas interesadas en participar en el proyecto de investigación, a saber:

- Que dentro del equipo de personas coinvestigadoras participe la persona encargada de cátedra o carrera.

- Disponer de al menos cinco horas quincenales para el trabajo con el equipo experto externo.

- Que la asignatura(s) objeto de estudio se ubique(n) en nivel de grado.

En atención a lo anterior, se conformaron seis equipos, que incluyen en mayor o menor medida, asignaturas de las cuatro escuelas (ver tabla 1).

Evaluar para Aprender: estado actual de catorce asignaturas en la Universidad Estatal a Distancia de Costa Rica Ana Cristina Umaña-Mata, Xinia Calvo-Cruz,

Natalia Salas-Quirós

DOI : http://dx.doi.org/10.22458/caes.v8i2.1809

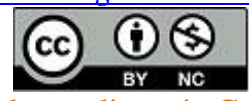


Tabla 1. Equipos de coinvestigación participantes

\begin{tabular}{|c|c|c|c|c|}
\hline Escuela & Carrera & $\begin{array}{c}\text { Asignatura } \\
\text { (objeto de } \\
\text { investigación }\end{array}$ & $\begin{array}{c}\text { Cátedra a la } \\
\text { que } \\
\text { pertenece la } \\
\text { asignatura(s) }\end{array}$ & $\begin{array}{c}\text { Personas } \\
\text { coinvestigadoras }\end{array}$ \\
\hline ECEN & $\begin{array}{l}\text { Ingeniería } \\
\text { agroindustrial }\end{array}$ & $\begin{array}{l}\text { Seminario de } \\
\text { desarrollo de } \\
\text { productos II }\end{array}$ & Agroindustria & $\begin{array}{l}\text { Carmen } \quad \text { Andrés } \\
\text { Jiménez } \\
\text { Sonia Vega Li }\end{array}$ \\
\hline ECSH & $\begin{array}{l}\text { Cátedra de } \\
\text { Historia }\end{array}$ & $\begin{array}{l}\text { Historia de la } \\
\text { Cultura }\end{array}$ & Historia & $\begin{array}{l}\text { Allyson Núñez } \\
\text { Lilliana Quesada } \\
\text { Martín }\end{array}$ \\
\hline ECSH & $\begin{array}{l}\text { Cátedra } \\
\text { Comunicación }\end{array}$ & $\begin{array}{l}\text { Comunicación Oral } \\
\text { y Escrita }\end{array}$ & $\begin{array}{l}\text { Comunicación } \\
\text { Oral y Escrita }\end{array}$ & $\begin{array}{l}\text { Rose Mary Mayorga } \\
\text { Celina Siles }\end{array}$ \\
\hline ECA & $\begin{array}{l}\text { Administración } \\
\text { de empresas } \\
\text { con énfasis en } \\
\text { Contaduría }\end{array}$ & $\begin{array}{l}\text { Estadística I } \\
\begin{array}{l}\text { Contabilidad de } \\
\text { costos I }\end{array} \\
\begin{array}{l}\text { Preparación } \\
\text { evaluación y } \\
\text { proyectos }\end{array}\end{array}$ & $\begin{array}{l}\text { Estadística } \\
\text { Contabilidad } \\
\text { Superior } \\
\text { Producción }\end{array}$ & $\begin{array}{l}\text { Eduardo Jirón } \\
\text { Alexandra Gómez } \\
\text { Elisa Sánchez }\end{array}$ \\
\hline
\end{tabular}

Fuente: elaboración del equipo

Evaluar para Aprender: estado actual de catorce asignaturas en la Universidad Estatal a

Distancia de Costa Rica

Ana Cristina Umaña-Mata, Xinia Calvo-Cruz,

Natalia Salas-Quirós

DOI : http://dx.doi.org/10.22458/caes.v8i2.1809

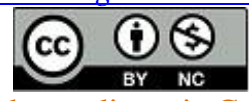


Tabla 1. Equipos de coinvestigación participantes (continuación)

\begin{tabular}{|c|c|c|c|c|}
\hline Escuela & Carrera & $\begin{array}{c}\text { Asignatura } \\
\text { (objeto de } \\
\text { investigación }\end{array}$ & $\begin{array}{c}\text { Cátedra a la } \\
\text { que } \\
\text { pertenece la } \\
\text { asignatura(s) }\end{array}$ & $\begin{array}{c}\text { Personas } \\
\text { coinvestigadoras }\end{array}$ \\
\hline ECE & $\begin{array}{l}\text { Administración } \\
\text { Educativa }\end{array}$ & $\begin{array}{l}\text { Administración de } \\
\text { la educación. } \\
\text { Planificación de la } \\
\text { Educación } \\
\text { Sociología de la } \\
\text { educación } \\
\text { Relación } \\
\text { Institución } \\
\text { educativa- } \\
\text { comunidad }\end{array}$ & $\begin{array}{l}\text { Estadística y } \\
\text { Administración } \\
\text { Educativa } \\
\text { Planificación } \\
\text { Educativa } \\
\\
\text { Educación } \\
\text { para el } \\
\text { Desarrollo }\end{array}$ & $\begin{array}{l}\text { Adrián Solano Castro } \\
\text { Ingrid Monge } \\
\text { Rodríguez } \\
\text { Gerardo Moreira } \\
\text { Ramírez } \\
\text { Margarita Jiménez } \\
\text { Romero }\end{array}$ \\
\hline ECE & $\begin{array}{l}\text { Educación } \\
\text { Primaria I y II } \\
\text { Ciclos }\end{array}$ & $\begin{array}{l}\text { Lectoescritura I y } \\
\text { II (modalidad } \\
\text { regular) } \\
\text { Lectoescritura I y } \\
\text { II (modalidad } \\
\text { virtual) }\end{array}$ & $\begin{array}{l}\text { Didáctica del } \\
\text { Lenguaje }\end{array}$ & $\begin{array}{l}\text { Jenny Bogantes } \\
\text { Pessoa }\end{array}$ \\
\hline
\end{tabular}

Fuente: elaboración del equipo

Evaluar para Aprender: estado actual de catorce asignaturas en la Universidad Estatal a

Distancia de Costa Rica

Ana Cristina Umaña-Mata, Xinia Calvo-Cruz,

Natalia Salas-Quirós

DOI : http://dx.doi.org/10.22458/caes.v8i2.1809

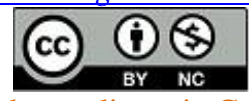




\subsection{Descripción de la etapa diagnóstico}

El diagnóstico es la primera etapa de la investigación acción. Se concreta con la identificación de una situación problema que se desea mejorar. En la etapa de diagnóstico se responde a la pregunta ¿Qué se va a investigar? El objetivo de la etapa diagnóstica de la presente investigación es el que se indica a continuación:

> Identificar el estado actual de las propuestas evaluativas de las asignaturas objeto de estudio, según el marco teórico-metodológico del evaluar para aprender.

Para la identificación del estado actual de cada una de las propuestas evaluativas, el equipo de investigación principal solicitó a los equipos coinvestigadores, consignar en una matriz aportada por el equipo principal, el detalle de cada asignatura, respecto a los siguientes datos generales: modalidad, regularidad de la oferta, número de estudiantes promedio por oferta, promedio de aprobación, así como su naturaleza.

Con respecto a cada forma evaluativa en particular, debían indicar: si era individual o grupal, interactiva o regular, que porcentaje tenía, el cálculo de horas de trabajo académico que demanda al estudiantado, el tipo de instrumento con el que se asignó la calificación, el nivel de participación de los actores (heteroevaluación, coevaluación y autoevaluación), indicar los medios de comunicación utilizados para la retroalimentación, así como el número de veces que se devolvía la entrega antes de asignar nota final, y finalmente, detallar el medio de entrega.

En otro orden de cosas, la misma matriz designaba una última columna para indicar las situaciones insatisfactorias de cada forma evaluativa, de acuerdo a los criterios e indicadores sobre las características y funciones de evaluar para aprender (ver tabla 2).

Evaluar para Aprender: estado actual de catorce asignaturas en la Universidad Estatal a

Distancia de Costa Rica

Ana Cristina Umaña-Mata, Xinia Calvo-Cruz,

Natalia Salas-Quirós

DOI : http://dx.doi.org/10.22458/caes.v8i2.1809

\section{(c) $($ ) $(8)$}


Tabla 2. Criterios e indicadores sobre características y funciones del evaluar para aprender

\begin{tabular}{|c|c|c|}
\hline Criterios & Indicadores & Sub indicadores \\
\hline \multirow{4}{*}{$\begin{array}{l}\text { 1.Presencia de } \\
\text { los niveles de } \\
\text { participación de } \\
\text { los actores } \\
\text { (docente } \\
\text { /docencia, } \\
\text { estudiante, } \\
\text { pares) }\end{array}$} & \multirow[t]{2}{*}{ Heteroevaluación } & $\begin{array}{l}\text { Informa al estudiante sobre el nivel de } \\
\text { aprendizaje alcanzado. }\end{array}$ \\
\hline & & $\begin{array}{l}\text { Establece lo que le falta al estudiante para } \\
\text { alcanzar el aprendizaje propuesto. }\end{array}$ \\
\hline & Coevaluación & $\begin{array}{l}\text { Evalúa habilidades para el trabajo } \\
\text { colaborativo (responsabilidad, liderazgo, } \\
\text { habilidades interpersonales, desarrollo } \\
\text { autónomo de la tarea). }\end{array}$ \\
\hline & Autoevaluación & $\begin{array}{l}\text { El estudiante revisa sus debilidades, } \\
\text { habilidades y fortalezas. }\end{array}$ \\
\hline \multirow{3}{*}{$\begin{array}{l}\text { 2. Presencia de } \\
\text { actividades } \\
\text { evaluativas } \\
\text { auténticas in situ } \\
\text { o simuladas. }\end{array}$} & \multirow[t]{2}{*}{$\begin{array}{l}\text { Actividades } \\
\text { evaluativas con } \\
\text { simulaciones }\end{array}$} & $\begin{array}{l}\text { Coherencia de las actividades evaluativas con } \\
\text { el contexto socio-profesional. }\end{array}$ \\
\hline & & \multirow[t]{2}{*}{ Las actividades evaluativas se realizan in situ. } \\
\hline & $\begin{array}{l}\text { Actividades } \\
\text { evaluativas } \\
\text { realizadas in situ }\end{array}$ & \\
\hline $\begin{array}{l}\text { 3.Presencia de } \\
\text { actividades } \\
\text { evaluativas } \\
\text { alternativas }\end{array}$ & $\begin{array}{l}\text { Actividades } \\
\text { (formas) } \\
\text { evaluativas } \\
\text { distintas a la } \\
\text { prueba escrita. }\end{array}$ & $\begin{array}{l}\text { Incluye niveles de participación de los actores } \\
\text { en la evaluación. }\end{array}$ \\
\hline \multirow{3}{*}{$\begin{array}{l}\text { 4. Organización } \\
\text { de trabajo } \\
\text { colaborativo } \\
\text { para el } \\
\text { desarrollo de } \\
\text { actividades } \\
\text { evaluativas. }\end{array}$} & \multirow{3}{*}{$\begin{array}{l}\text { Búsqueda de una } \\
\text { meta u objetivo en } \\
\text { común. } \\
\text { Intercomunicación } \\
\text { y consenso. } \\
\text { (García, } 2014 \text {, p. } \\
\text { 180) }\end{array}$} & $\begin{array}{l}\text { Potencia habilidades de análisis, síntesis, } \\
\text { argumentación y valoración. }\end{array}$ \\
\hline & & $\begin{array}{l}\text { Señala al estudiante las habilidades } \\
\text { interpersonales a desarrollar. }\end{array}$ \\
\hline & & Retroalimenta el desarrollo de la tarea. \\
\hline
\end{tabular}

Evaluar para Aprender: estado actual de catorce asignaturas en la Universidad Estatal a Distancia de Costa Rica Ana Cristina Umaña-Mata, Xinia Calvo-Cruz, Natalia Salas-Quirós DOI : http://dx.doi.org/10.22458/caes.v8i2.1809 


\begin{tabular}{|c|c|c|}
\hline \multirow{4}{*}{$\begin{array}{l}\text { Criterios } \\
\text { 5. Utilización de } \\
\text { instrumentos de } \\
\text { la cultura. }\end{array}$} & Indicadores & Sub indicadores \\
\hline & \multirow{3}{*}{$\begin{array}{l}\text { Instrumentos y } \\
\text { herramientas } \\
\text { tecnológicas } \\
\text { propias de la } \\
\text { disciplina y del } \\
\text { contexto socio- } \\
\text { profesional. }\end{array}$} & Utiliza correctamente el lenguaje disciplinar. \\
\hline & & Utiliza metodologías propias de la disciplina. \\
\hline & & $\begin{array}{l}\text { Utiliza apoyo tecnológico propio de la } \\
\text { disciplina. }\end{array}$ \\
\hline \multirow{5}{*}{$\begin{array}{l}\text { Criterios } \\
6 \text { 6. Utilización de } \\
\text { lo cuantificable } \\
\text { como } \\
\text { procedimiento } \\
\text { final de la } \\
\text { actividad } \\
\text { evaluativa. }\end{array}$} & Indicadores & Sub indicadores \\
\hline & \multirow{4}{*}{$\begin{array}{l}\text { Validez técnica } \\
\text { de los } \\
\text { instrumentos }\end{array}$} & $\begin{array}{l}\text { Incluye criterios e indicadores de evaluación } \\
\text { (cuantitativos y cualitativos). }\end{array}$ \\
\hline & & $\begin{array}{l}\text { Los criterios de evaluación son coherentes } \\
\text { con la actividad evaluativa. }\end{array}$ \\
\hline & & $\begin{array}{l}\text { La escala de calificación permite valorar de } \\
\text { forma válida el criterio. }\end{array}$ \\
\hline & & $\begin{array}{l}\text { Evalúa todo el contenido, según los objetivos } \\
\text { de la actividad. }\end{array}$ \\
\hline \multirow{4}{*}{$\begin{array}{l}\text { 7. Relación de la } \\
\text { actividad } \\
\text { evaluativa con el } \\
\text { objetivo (s) de } \\
\text { aprendizaje. }\end{array}$} & \multirow{2}{*}{$\begin{array}{l}\text { La actividad } \\
\text { evaluativa } \\
\text { (técnica o } \\
\text { estrategia) } \\
\text { permite obtener } \\
\text { evidencias del } \\
\text { logro del } \\
\text { aprendizaje. }\end{array}$} & $\begin{array}{l}\text { La técnica utilizada permite obtener } \\
\text { evidencias del logro del aprendizaje. }\end{array}$ \\
\hline & & $\begin{array}{l}\text { La estrategia permite obtener evidencias del } \\
\text { logro del aprendizaje. }\end{array}$ \\
\hline & \multirow{2}{*}{$\begin{array}{l}\text { Pertinencia del } \\
\text { medio para } \\
\text { alcanzar el logro } \\
\text { de aprendizaje } \\
\text { objeto de la } \\
\text { actividad } \\
\text { evaluativa }\end{array}$} & $\begin{array}{l}\text { La herramienta seleccionada de la plataforma } \\
\text { virtual para la actividad evaluativa es } \\
\text { pertinente para alcanzar el logro de } \\
\text { aprendizaje objeto de la actividad evaluativa. }\end{array}$ \\
\hline & & $\begin{array}{l}\text { El medio (visual, audio, audiovisual, sistema } \\
\text { de comunicación) seleccionado para la } \\
\text { actividad evaluativa es pertinente para } \\
\text { alcanzar el logro de aprendizaje objeto de la } \\
\text { actividad evaluativa. }\end{array}$ \\
\hline
\end{tabular}

Evaluar para Aprender: estado actual de catorce asignaturas en la Universidad Estatal a Distancia de Costa Rica

Ana Cristina Umaña-Mata, Xinia Calvo-Cruz, Natalia Salas-Quirós

DOI : http://dx.doi.org/10.22458/caes.v8i2.1809

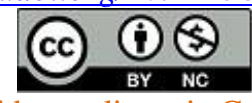




\begin{tabular}{|c|c|c|}
\hline \multirow[t]{7}{*}{ Criterios } & Indicadores & Sub indicadores \\
\hline & $\begin{array}{l}\text { Suficiencia de las } \\
\text { instrucciones } \\
\text { (procedimientos) } \\
\text { de la actividad } \\
\text { evaluativa para } \\
\text { alcanzar el logro } \\
\text { de aprendizaje. }\end{array}$ & $\begin{array}{l}\text { La actividad evaluativa presenta la siguiente } \\
\text { estructura: información administrativa, objetivo } \\
\text { de aprendizaje y objetivo de la actividad, } \\
\text { medio en que se realiza, tipo de instrumento } \\
\text { de evaluación, si es grupal o individual, } \\
\text { instrucciones generales y específicas. }\end{array}$ \\
\hline & $\begin{array}{l}\text { Coherencia con el } \\
\text { nivel de } \\
\text { conocimiento }\end{array}$ & $\begin{array}{l}\text { La actividad evaluativa es coherente con el } \\
\text { nivel de conocimiento establecido en el } \\
\text { objetivo de aprendizaje. }\end{array}$ \\
\hline & $\begin{array}{l}\text { Coherencia con el } \\
\text { tipo de } \\
\text { conocimiento } \\
\text { (conceptual, } \\
\text { procedimental y } \\
\text { actitudinal) }\end{array}$ & $\begin{array}{l}\text { La actividad evaluativa es coherente con el } \\
\text { tipo de conocimiento (conceptual, } \\
\text { procedimental y actitudinal) establecido en el } \\
\text { objetivo de aprendizaje. }\end{array}$ \\
\hline & $\begin{array}{l}\text { Coherencia con el } \\
\text { contenido }\end{array}$ & $\begin{array}{l}\text { La actividad evaluativa es coherente con el } \\
\text { contenido establecido en el objetivo de la } \\
\text { actividad en relación con el objetivo de } \\
\text { aprendizaje. }\end{array}$ \\
\hline & & $\begin{array}{l}\text { La actividad evaluativa enfoca los aspectos } \\
\text { esenciales del contenido. }\end{array}$ \\
\hline & $\begin{array}{l}\text { Relación entre el } \\
\text { porcentaje } \\
\text { asignado a la } \\
\text { actividad } \\
\text { evaluativa y la } \\
\text { cantidad de } \\
\text { trabajo } \\
\text { académico que } \\
\text { ésta requiere. }\end{array}$ & $\begin{array}{l}\text { El porcentaje asignado a la actividad } \\
\text { evaluativa es proporcional a la cantidad de } \\
\text { trabajo académico que ésta requiere. }\end{array}$ \\
\hline $\begin{array}{l}\text { 8. Regulación del } \\
\text { proceso de } \\
\text { aprendizaje. }\end{array}$ & $\begin{array}{l}\text { Retroalimentación } \\
\text { de los procesos } \\
\text { de aprendizaje }\end{array}$ & $\begin{array}{l}\text { La retroalimentación que se brinda le permite } \\
\text { al estudiantado detectar errores, debilidades y } \\
\text { aciertos para el logro del aprendizaje } \\
\text { propuesto. }\end{array}$ \\
\hline
\end{tabular}

Evaluar para Aprender: estado actual de catorce asignaturas en la Universidad Estatal a Distancia de Costa Rica

Ana Cristina Umaña-Mata, Xinia Calvo-Cruz,

Natalia Salas-Quirós

DOI : http://dx.doi.org/10.22458/caes.v8i2.1809

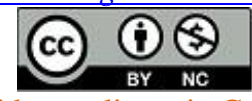




\begin{tabular}{|c|c|c|}
\hline \multirow[t]{5}{*}{ Criterios } & Indicadores & Sub indicadores \\
\hline & \multirow[t]{2}{*}{$\begin{array}{l}\text { (actividades } \\
\text { evaluativas) }\end{array}$} & $\begin{array}{l}\text { La retroalimentación se brinda en el momento } \\
\text { oportuno para mejorar los procesos de } \\
\text { aprendizaje. }\end{array}$ \\
\hline & & $\begin{array}{l}\text { Se brinda la oportunidad al estudiantado para } \\
\text { evidenciar la mejora de la actividad evaluativa } \\
\text { a partir de la retroalimentación dada. }\end{array}$ \\
\hline & $\begin{array}{l}\text { Suficiencia de la } \\
\text { información inicial } \\
\text { que requiere el } \\
\text { estudiantado. }\end{array}$ & $\begin{array}{l}\text { El documento de Orientaciones Académicas y } \\
\text { las instrucciones de las actividades } \\
\text { evaluativas dan la información suficiente para } \\
\text { orientar al estudiantado en el logro de los } \\
\text { objetivos de aprendizaje propuestos. }\end{array}$ \\
\hline & $\begin{array}{l}\text { Propicia la } \\
\text { relación de lo } \\
\text { cognitivo con lo } \\
\text { emocional. }\end{array}$ & $\begin{array}{l}\text { El proceso de regulación del aprendizaje } \\
\text { propicia en el estudiantado las actitudes de } \\
\text { reflexión de su propio proceso de aprendizaje. }\end{array}$ \\
\hline \multirow{3}{*}{$\begin{array}{l}9 . \\
\text { Autorregulación } \\
\text { del aprendizaje } \\
\text { como evidencia } \\
\text { de aprender a } \\
\text { aprender. }\end{array}$} & \multirow{3}{*}{$\begin{array}{l}\text { La mediación } \\
\text { pedagógica } \\
\text { propicia el } \\
\text { desarrollo de los } \\
\text { saberes } \\
\text { metacognitivos en } \\
\text { el estudiantado. }\end{array}$} & $\begin{array}{l}\text { La mediación pedagógica permite al } \\
\text { estudiantado detectar cuándo aprende. }\end{array}$ \\
\hline & & $\begin{array}{l}\text { La mediación pedagógica permite al } \\
\text { estudiantado detectar que ha aprendido y lo } \\
\text { que no. }\end{array}$ \\
\hline & & $\begin{array}{l}\text { La mediación pedagógica permite al } \\
\text { estudiantado detectar lo que requiere para } \\
\text { aprender. }\end{array}$ \\
\hline
\end{tabular}

Fuente: Jiménez, L. (2015). Criterios e indicadores sobre características y funciones del evaluar para aprender. Programa de Apoyo Curricular y Evaluación de los Aprendizajes

Es importante indicar que el equipo de investigación del PACE, se distribuyó los equipos coinvestigadores, con el fin de orientar y guiar hacia la consecución del diagnóstico de cada asignatura. Esta etapa demandó mucho análisis y revisión de cada forma evaluativa

Evaluar para Aprender: estado actual de catorce asignaturas en la Universidad Estatal a Distancia de Costa Rica Ana Cristina Umaña-Mata, Xinia Calvo-Cruz, Natalia Salas-Quirós

DOI : http://dx.doi.org/10.22458/caes.v8i2.1809

\section{(c) $\underset{\mathrm{BY}}{\mathrm{BC}}(\mathrm{NC}$}


en un afán de contrarrestar con los criterios e indicadores dados, e identificar insatisfacciones desde el marco teórico -metodológico de evaluar para aprender.

En este mismo ciclo de diagnóstico, mientras cada equipo trabajaba en su asignatura, el equipo del PACE planificó algunos espacios formativos, cuyo objetivo fue que las personas participantes adquirieran conocimientos básicos para la comprensión del tema en torno al que gira la investigación. Finalmente, en la etapa de diagnóstico, cada equipo de coinvestigación en coordinación con la investigadora asesora del PACE realizó un análisis de las causas y efectos identificados, producto de las insatisfacciones de la evaluación de los aprendizajes de su asignatura desde el marco de evaluar para aprender; lo anterior, con el fin de seleccionar las que serían atendidas con intenciones de mejora en este primer ciclo de investigación acción. Aunado a lo anterior, se hizo la selección de los campos de acción que sustentarán las hipótesis sobre las cuales se realizará el planteamiento del plan de acción.

\section{Resultados del diagnóstico}

\section{- Resultados por criterio}

$>$ Presencia de los niveles de participación de los actores (docente/docencia, estudiante, pares)

La poca presencia de los niveles de participación de los actores se marcó fuertemente en las catorce asignaturas en estudio. De los tres indicadores de este criterio, la heteroevaluación fue el que tuvo un menor número de negativos; en este caso cinco asignaturas indicaron que no hay presencia de este nivel de participación en las formas evaluativas de sus asignaturas; igual número de asignaturas asumieron que sí tienen presencia parcial de este nivel de participación. Mientras que, según los datos arrojados

Evaluar para Aprender: estado actual de catorce asignaturas en la Universidad Estatal a

Distancia de Costa Rica

Ana Cristina Umaña-Mata, Xinia Calvo-Cruz,

Natalia Salas-Quirós

DOI : http://dx.doi.org/10.22458/caes.v8i2.1809

\section{(c) ${ }_{\mathrm{BY}}(2)$}


del diagnóstico, cuatro asignaturas sí consideraron este nivel de participación. Sobre la heteroevaluación, se destaca el caso de la asignatura de Seminario de Desarrollo de Productos II, en la cual se indica que sí estuvo presente, porque el profesor asigna un puntaje final a cada forma evaluativa, pero el estudiantado no conoce el nivel de logro ni lo que falta por lograr, igual observación se hace para las asignaturas de Estadística I, Contabilidad de costos I y Preparación y Evaluación de Proyectos, quienes indican que asumen este nivel de participación, pero no se le establece al estudiantado lo que falta por aprender.

Finalmente, en este nivel de participación destacaron las asignaturas Lectoescritura I y II en modalidad virtual, respecto al uso de matrices de evaluación para algunas actividades.

Sobre este criterio, llaman la atención los resultados relacionados con la coevaluación, siendo este el nivel de participación, en el cual se marca mayoritariamente la ausencia en las asignaturas en estudio. Sobre este particular, 12 asignaturas indicaron su ausencia total, mientras que únicamente Lectoescritura I y Lectoescritura II, ambas ofertadas en modalidad virtual, sí asumieron este nivel de participación de acuerdo a lo establecido en el marco teórico metodológico de evaluar para aprender, mientras que la misma asignatura en modalidad regular no asumió la coevaluación.

Otro dato de importancia refiere al nivel de participación autoevaluación, en el cual los resultados arrojaron 11 respuestas negativas respecto a la ausencia de este indicador en las asignaturas en estudio, mientras que únicamente tres indicaron que este nivel de participación estuvo parcialmente presente, contrario a lo estipulado en el Modelo Pedagógico Institucional.

Evaluar para Aprender: estado actual de catorce asignaturas en la Universidad Estatal a

Distancia de Costa Rica

Ana Cristina Umaña-Mata, Xinia Calvo-Cruz,

Natalia Salas-Quirós

DOI : http://dx.doi.org/10.22458/caes.v8i2.1809

\section{(c) $($ ) $(8)$}


Sobre este nivel se destaca que las asignaturas Estadística I, Contabilidad de Costos I y Preparación y Evaluación de proyectos contaron con ejercicios de autoevaluación en los materiales didácticos, así como en la plataforma virtual. En el caso de Contabilidad de Costos I, contó con un instrumento adicional para la intención de autoevaluación; sin embargo, faltó incorporar la revisión del estudiante de sus debilidades, habilidades y fortalezas.

\section{> Presencia de actividades evaluativas auténticas in situ o simuladas}

En este criterio se analizaron dos indicadores, a saber: actividades evaluativas con simulaciones y actividades evaluativas realizadas in situ. Sobre las actividades evaluativas con simulaciones, el resultado del diagnóstico suma nueve presencias de este indicador como parte de las actividades evaluativas de las asignaturas en estudio, mientras que solo cinco asignaturas reportan ausencia de este indicador. Al respecto, cabe señalar que la asignatura Seminario de Desarrollo de Productos II realizó un proyecto en una planta piloto- CITA-UCR, sin embargo, el instrumento de evaluación carece de criterios, indicadores y escala de calificación validos técnicamente.

Sobre la presencia o ausencia de actividades evaluativas realizadas in situ, se obtiene como resultado que nueve asignaturas no las consideraron dentro de sus actividades evaluativas y cinco de ellas sí las contemplaron. Estas últimas son: Lectoescritura I y II en las modalidades regular y virtual y Seminario de Desarrollo de Productos II. Otro aspecto que llama la atención es que las asignaturas Estadística I, Contabilidad de Costos I y Preparación y Evaluación de Proyectos, no contaron con este indicador, por cuanto estas actividades podrían implicar un riesgo para el trabajo de empresa u organización donde podría llevarse a cabo.

Evaluar para Aprender: estado actual de catorce asignaturas en la Universidad Estatal a

Distancia de Costa Rica

Ana Cristina Umaña-Mata, Xinia Calvo-Cruz,

Natalia Salas-Quirós

DOI : http://dx.doi.org/10.22458/caes.v8i2.1809

\section{(c) $\underset{\mathrm{BY}}{\mathrm{Br}}(\mathrm{SC}$}




\section{> Presencia de actividades evaluativas alternativas}

Con respecto al criterio relacionado con la ausencia o presencia de formas evaluativas distintas a la prueba escrita, es decir, alternativas, se obtiene que de las catorce asignaturas ninguna negó tener formas alternativas, todas lo asumieron dentro de sus formas evaluativas como mínimo de manera parcial. En detalle siete asignaturas indican que sí tienen formas alternativas y las otras siete recurren parcialmente a formas diferentes a la pruebe escrita. Sobre este particular se destaca que la asignatura de Comunicación Oral y Escrita hizo referencia a la práctica evaluativa, citando entre las formas alternativas un proyecto y una tarea. Mientras que Historia de la Cultura utilizó el portafolio, el álbum y el ensayo; esta última asignatura ronda un aproximado de 1400 estudiantes.

Un aspecto importante en este criterio es que, en el caso de las asignaturas de la Escuela de Ciencias de la Educación se indica que, a pesar de que se presenta alguna actividad que sea alternativa a la prueba escrita, estas carecían de información de retorno y del respectivo instrumento de evaluación. En este mismo criterio, las asignaturas que corresponden a la Escuela de Ciencias de la Administración, que, si bien es cierto, presentaban actividades evaluativas diversas y distintas a la prueba escrita, estas no contemplaban los niveles de autoevaluación y coevaluación. En el caso de la asignatura que representa la Escuela de Ciencias Exactas y Naturales, se indicó que sí había otras formas evaluativas diferentes a la prueba escrita, que las mismas carecen de participación de los actores.

Evaluar para Aprender: estado actual de catorce asignaturas en la Universidad Estatal a

Distancia de Costa Rica

Ana Cristina Umaña-Mata, Xinia Calvo-Cruz,

Natalia Salas-Quirós

DOI : http://dx.doi.org/10.22458/caes.v8i2.1809

\section{(c) $(1)(2)$}




\section{$>$ Organización de trabajo colaborativo para el desarrollo de actividades evaluativas.}

Respecto a éste se tiene que dos de las catorce asignaturas objeto de estudio sí cumplen con este criterio, seis lo cumplen de forma parcial y seis no lo cumplen.

En el caso de Seminario de Desarrollo de Productos II y Preparación y Evaluación de Proyectos se tiene trabajo en grupos, pero no se cumple con las características del trabajo colaborativo, según lo propuesto por García (2014), a saber: interdependencia positiva, responsabilidad en el aprendizaje y liderazgo compartido, se enseñan habilidades interpersonales, el rol docente es de observación y retroalimentación de la tarea, así como la importancia tanto del proceso como del producto. Es decir, en estas dos no se tiene la retroalimentación en el desarrollo de la tarea, o no existe un proceso de coevaluación (responsabilidad en el aprendizaje compartido).

En el caso de las asignaturas Estadística I, Contabilidad de Costos I y Preparación y Evaluación de Proyectos se incluyen actividades evaluativas que potencian el análisis, la síntesis y la argumentación, que son habilidades básicas para el trabajo colaborativo; sin embargo, no se señalan al estudiantado las habilidades interpersonales a desarrollar.

De las seis asignaturas que no presentan trabajo colaborativo se tiene que una de ellas lo justifican por ser de modalidad regular a distancia, es decir, hacen uso de los medios tradicionales de la educación a distancia de la UNED para la mediación pedagógica; mientras que en Estadística I no se lleva a cabo por tener una matrícula masiva durante cada cuatrimestre que se oferta, lo que podría implicar el requerir una mayor cantidad de

Evaluar para Aprender: estado actual de catorce asignaturas en la Universidad Estatal a

Distancia de Costa Rica

Ana Cristina Umaña-Mata, Xinia Calvo-Cruz,

Natalia Salas-Quirós

DOI : http://dx.doi.org/10.22458/caes.v8i2.1809

\section{(c) $($ ) $(8)$}


profesores revisores. Las demás asignaturas, solamente, presentan trabajos individuales o pruebas escritas como formas de evaluación.

\section{$>$ Utilización de instrumentos de la cultura.}

Respecto a este criterio, se tiene que la mayoría de las asignaturas objeto de estudio (10 asignaturas) hacen uso de instrumentos y herramientas tecnológicas propias de la disciplina y el contexto socioprofesional; mientras que dos lo hacen parcialmente y dos no lo hacen.

Así, se tiene que todas las asignaturas de la ECE sí cumplen este criterio, además de las asignaturas Estadística I y Preparación y Evaluación de Proyectos. Sin embargo, en la asignatura Seminario de Desarrollo de Productos II se carece de análisis de casos, una metodología muy propia del desarrollo de productos; mientras que en el caso de Contabilidad de Costos I existen dificultades en el uso del lenguaje disciplinar por cuanto el libro que se utiliza es de editorial extrajera con términos que no son de uso en el país. Además, se tiene términos propios de contabilidad que en otras asignaturas se enseñan con otro nombre.

\section{$>$ Utilización de lo cuantificable como procedimiento final de la actividad evaluativa.}

De acuerdo con la información recopilada sobre los tipos de instrumentos de evaluación, la mayor parte de las asignaturas objeto de estudio hacen uso de la prueba escrita como instrumento de evaluación (ocho asignaturas), seguido de la matriz de valoración o rúbrica (siete asignaturas). Asimismo, la escala de calificación, la guía de evaluación (según la

Evaluar para Aprender: estado actual de catorce asignaturas en la Universidad Estatal a

Distancia de Costa Rica

Ana Cristina Umaña-Mata, Xinia Calvo-Cruz,

Natalia Salas-Quirós

DOI : http://dx.doi.org/10.22458/caes.v8i2.1809

\section{(c) $(1)(2)$}


plataforma Moodle), el cuestionario y la lista de cotejo son nombrados solo una vez cada uno.

De estos, se tiene que las asignaturas Historia de la Cultura y Comunicación Oral y Escrita sí cumplen con la validez técnica de los instrumentos de evaluación utilizados, mientras que las asignaturas de la ECA junto con Lectoescritura I y II, en sus dos modalidades de oferta, lo cumplen parcialmente. De las asignaturas que no cumplen o cumplen parcialmente este criterio, se evidencia dificultades en la estructuración de los instrumentos.

Para el caso del Seminario de Desarrollo de Productos II, en los instrumentos de evaluación se evidencian básicamente criterios de tipo cuantitativo; además, existe una discrepancia entre la relación de horas indicadas desde el diseño curricular y las actividades evaluativas desarrolladas.

En las asignaturas objeto de estudio de la ECA se presentan instrumentos de evaluación, según el tipo de actividad evaluativa, sin embargo, en el caso de las matrices de valoración o rúbricas que se utilizan requieren de mejoras en cuanto a su planteamiento. Sin embargo, en las pruebas escritas, se cuenta con la asesoría de la especialista de la escuela, con lo que se evidencian mejoras en su estructura.

\section{$>$ Relación de la actividad evaluativa con el objetivo (s) de aprendizaje.}

Este criterio contempla los siguientes indicadores: a) la actividad evaluativa permite obtener evidencias del logro del aprendizaje, b) pertinencia del medio para alcanzar el

Evaluar para Aprender: estado actual de catorce asignaturas en la Universidad Estatal a

Distancia de Costa Rica

Ana Cristina Umaña-Mata, Xinia Calvo-Cruz,

Natalia Salas-Quirós

DOI : http://dx.doi.org/10.22458/caes.v8i2.1809

\section{(c) $\underset{\mathrm{BY}}{\mathrm{Br}}(\mathrm{SC}$}


logro, c) suficiencia de las instrucciones de la actividad evaluativa para alcanzar el logro de aprendizaje, d) coherencia con el nivel del conocimiento, e) coherencia con el tipo de conocimiento, f) coherencia con el contenido, g) relación entre el porcentaje asignado a la actividad evaluativa y la cantidad de trabajo académico que se requiere.

Se encuentra que, la carencia de evidencias sobre el logro del aprendizaje está presente en siete asignaturas, mientras que en seis sí las hay. En la asignatura denominada Preparación y Evaluación de Proyectos, solamente se tienen registros parciales del logro del aprendizaje.

Por otra parte, en las asignaturas Seminario de Desarrollo de Productos II, Sociología de la Educación y Relación Institución Educativa Comunidad se identifica que de manera parcial el medio utilizado permite alcanzar el logro del aprendizaje, mientras en ocho de las asignaturas en estudio reportan que el medio permite alcanzar el logro de aprendizaje $\mathrm{y}$, solamente en tres no se logra. En estos últimos casos se trata de asignaturas que se ofertan de forma regular, donde los medios no son utilizados.

La suficiencia de las instrucciones de la actividad evaluativa para alcanzar el logro del aprendizaje está presente en siete de las asignaturas, mientras que en cuatro de ellas no. En el caso de las asignaturas Sociología de la Educación, Relación Institución Educativa Comunidad y Seminario de Desarrollo de Productos II las instrucciones se presentan de forma parcial.

En relación con la coherencia con el nivel del conocimiento, en el caso de las asignaturas en estudio, se encuentra que de las catorce, en seis de ellas se evidencia este tipo de

Evaluar para Aprender: estado actual de catorce asignaturas en la Universidad Estatal a

Distancia de Costa Rica

Ana Cristina Umaña-Mata, Xinia Calvo-Cruz,

Natalia Salas-Quirós

DOI : http://dx.doi.org/10.22458/caes.v8i2.1809

\section{(c) $($ ) $(8)$}


coherencia, en otras seis dicha relación es de tipo parcial, mientras que en las asignaturas Seminario de Desarrollo de Productos II y Planificación de la Educación, este criterio no se presenta. Por otra parte, la coherencia con el tipo de conocimiento desarrollado en las actividades evaluativas, según lo propuesto en el diseño curricular de las asignaturas; en el grupo en estudio hay siete asignaturas que cumplen con esta condición, cinco de las catorce lo evidencian de forma parcial, mientras que en el caso de las asignaturas de Planificación de la Educación y Administración de la Educación está totalmente ausente.

De acuerdo al criterio que refiere a la coherencia del contenido de las actividades evaluativas, se encontró en el diagnóstico que en cinco de las asignaturas se cumple esta condición. En seis de las catorce asignaturas se cumple de manera parcial, mientras que en las asignaturas de Planificación de la Educación y Administración educativa no se cumple.

La relación entre el porcentaje asignado a las actividades evaluativas y la cantidad de trabajo académico que se requiere es un indicador considerado en seis de las asignaturas, mientras que en otras seis de ellas es considerado de forma parcial. En las asignaturas Administración de la Educación y Seminario de Desarrollo de Productos II no es un indicador que sea tomado en cuenta en el proceso evaluativo.

\section{$>$ Regulación del proceso de aprendizaje}

En este criterio fueron analizados tres indicadores a saber: a) retroalimentación de los procesos de aprendizaje, b) suficiencia de la información inicial que requiere el estudiantado y c) la relación que se propicia entre los componentes cognitivo y lo emocional en el estudiantado. Con respecto a la inclusión de la retroalimentación en los

Evaluar para Aprender: estado actual de catorce asignaturas en la Universidad Estatal a

Distancia de Costa Rica

Ana Cristina Umaña-Mata, Xinia Calvo-Cruz,

Natalia Salas-Quirós

DOI : http://dx.doi.org/10.22458/caes.v8i2.1809

\section{(c) $($ ) $(8)$}


procesos de aprendizaje, seis de las asignaturas en estudio reportaron que se implementa de forma parcial, donde se evidencia que en la mayoría de estos casos es necesario el mejoramiento de la misma. Por otra parte, en cuatro de las asignaturas no se contempla este indicador y en las asignaturas Lectoescritura I (regular), Lectoescritura I (virtual), Lectoescritura II (regular) y Lectoescritura II (virtual) si se llevan a cabo procesos de retroalimentación.

Con respecto al indicador referido a la suficiencia de la información inicial que es requerida por el estudiantado para el desarrollo de sus diversas actividades, se encuentra que en nueve de las asignaturas está presente, estando, en mayor medida, presente en las orientaciones académicas que se le otorgan al inicio del periodo lectivo a cada estudiante. En las asignaturas de Sociología de la Educación y Relación Institución Educativa Comunidad la información que se brinda cumple, parcialmente, con el criterio de suficiencia, mientras que, en las asignaturas de Planificación de la Educación, Administración de la Educación y Seminario de Desarrollo de Productos II no se cumple con la suficiencia de información requerida.

Finalmente, se encuentra que en la mayoría de las asignaturas hay poca o ninguna evidencia de la existencia de procesos de regulación del aprendizaje. En siete de las asignaturas no hay evidencia de que en este tipo de procesos de regulación de los aprendizajes se relacionen los componentes cognitivos con los emocionales en el desarrollo del proceso de aprendizaje del estudiantado. En cuatro de ellas se encuentra que no se establece, mientras que, en las asignaturas de Planificación de la Educación, Administración de la Educación y Seminario de Desarrollo de Productos II la relación entre los componentes se da de manera parcial.

Evaluar para Aprender: estado actual de catorce asignaturas en la Universidad Estatal a

Distancia de Costa Rica

Ana Cristina Umaña-Mata, Xinia Calvo-Cruz,

Natalia Salas-Quirós

DOI : http://dx.doi.org/10.22458/caes.v8i2.1809

\section{(c) $($ ) $(8)$}




\section{Conclusiones}

- Los hallazgos del diagnóstico evidencian la necesidad de la existencia de un marco teórico-metodológico de evaluar para aprender en la universidad que permita orientar los procesos de evaluación institucionales.

- A partir de la información recopilada en este diagnóstico se puede decir que las personas participantes en la investigación (equipo de coinvestigación) desconocen el marco teórico- metodológico de evaluar para aprender, lo que ocasionó en algunos casos dificultades para la comprensión de las implicaciones de cada criterio relacionado con las características y funciones del evaluar para aprender, lo que a su vez acarreo dificultades para identificar las insatisfacciones en las asignaturas de cada equipo.

- Se evidencia el uso de estrategias y técnicas alternativas de evaluación. Sin embargo, el diagnóstico evidencia que hay desconocimiento de elementos técnicos que se deben tomar en cuenta en su construcción e implementación.

- El ejercicio de diagnóstico de las asignaturas evidencia que la prueba escrita mantiene un lugar predominante en la evaluación de los aprendizajes, en detrimento de opciones alternativas que pueden acogerse desde las realidades de las asignaturas.

- Según los resultados del diagnóstico, las formas evaluativas alternativas son más evidentes en las asignaturas de modalidad virtual o híbrida, así como en aquellas con un número de estudiantes no considerado "masivo".

- A pesar de que cada equipo de coinvestigación identificó diversas problemáticas en sus asignaturas, es común que se indiquen como causas reiterativas, la poca retroalimentación en los procesos de aprendizaje (y sus implicaciones), así como la validez técnica de los instrumentos utilizados en sus formas evaluativas.

Evaluar para Aprender: estado actual de catorce asignaturas en la Universidad Estatal a

Distancia de Costa Rica

Ana Cristina Umaña-Mata, Xinia Calvo-Cruz,

Natalia Salas-Quirós

DOI : http://dx.doi.org/10.22458/caes.v8i2.1809

\section{(c) $(1)(2)$}


- Se hace evidente la poca presencia de los niveles de participación de los actores en el proceso de mediación pedagógica.

- En general, todas las asignaturas reportan carencias importantes en la recolección de evidencias respecto al logro del aprendizaje de cada estudiante.

- El trabajo colaborativo no se presenta en las asignaturas diagnosticadas, por el frecuente uso de trabajo individualizados, o porque los trabajos se desarrollan con una metodología grupal distinta a la requerida por un proceso colaborativo, lo que, a su vez, propicia el bajo uso de la coevaluación.

- Existe una marcada tendencia hacia la evaluación final, pese a que en algunas asignaturas se da la posibilidad de entregar un mismo trabajo en varios avances, por lo que sigue teniendo mayor peso la calificación ante las funciones formativa y formadora de la evaluación.

\section{Recomendaciones}

- A partir de los resultados obtenidos se requiere de la priorización de aquellas acciones que se analicen como viables de implementación en las posteriores etapas de esta investigación-acción, a fin de determinar las principales mejoras que pueden ser llevadas a cabo desde el propio contexto de cada asignatura objeto de estudio.

- A nivel de universidad es necesario retomar los espacios de formación y capacitación sobre evaluación orientada hacia aprendizaje; que unidos a los procesos de asesoría curricular permitan la comprensión de su marco teórico-metodológico y faciliten su puesta en práctica en la oferta académica.

- Desde el PACE es recomendable continuar con los procesos de asesoría en materia de evaluación de los aprendizajes a partir de los criterios construidos sobre evaluar

Evaluar para Aprender: estado actual de catorce asignaturas en la Universidad Estatal a

Distancia de Costa Rica

Ana Cristina Umaña-Mata, Xinia Calvo-Cruz,

Natalia Salas-Quirós

DOI : http://dx.doi.org/10.22458/caes.v8i2.1809

\section{(c) $\underset{\mathrm{BY}}{\mathrm{Br}}(\mathrm{SC}$}


para aprender, con el propósito de favorecer espacios de análisis de cada asignatura acordes con sus características académicas y de la población que atiende.

\section{Referencias}

Bordas, M.I. y Cabrera, F. (enero-abril, 2001). Estrategias de evaluación de los aprendizajes centrados en el proceso. Revista Española de Pedagogía, LIX(218), $25-48$

Colmenares, A. M. (2012). Los aprendizajes en entornos virtuales evaluados bajo la concepción formadora. REIFOP, 15(1), 125-134. Recuperado de http//www.aufop.com

Colás, M.P. y Buendía, L. (1998). Investigación educativa. Sevilla, España: Editores Alfar.

Monereo, C., y Badia, A. (2013). Aprendizaje estratégico y tecnologías de la información y la comunicación: una revisión crítica. Revista teoría de la educación. Educación y cultura en la sociedad de la información, 14(2), 15-41.

Sacristán, J.G. (comp.) (2010). Saberes e incertidumbres sobre el curriculum. Madrid, España: Ediciones Morata.

Popham, J. (2014). La evaluación transformativa. El poder transformador de la evaluación formativa. Madrid, España: Narcea Ediciones.

Pozo, J. I. y Monereo, C. (coord.) (2000). El aprendizaje estratégico. Enseñar a aprender desde el currículo. Madrid, España: Aula XXI Santillana.

Universidad Estatal a Distancia (2004). Modelo pedagógico. San José, Costa Rica. UNED.

Universidad Estatal a Distancia (2012). Reglamento general estudiantil. San José, Costa Rica: UNED.

Evaluar para Aprender: estado actual de catorce asignaturas en la Universidad Estatal a

Distancia de Costa Rica

Ana Cristina Umaña-Mata, Xinia Calvo-Cruz,

Natalia Salas-Quirós

DOI : http://dx.doi.org/10.22458/caes.v8i2.1809

\section{(c) $(1)$ (8)}


Universidad Estatal a Distancia (2012). Informe sobre el proceso de consulta mediante la implementación de mesas temáticas para la construcción de los fundamentos para el plan académico UNED 2012-2017. San José, Costa Rica: UNED.

Universidad Estatal a Distancia. (2010). Reglamento de gestión académica de la Universidad Estatal a Distancia (2. ${ }^{\mathrm{a}}$ ed.). San José, Costa Rica: UNED.

Universidad Estatal a Distancia. (2013). Plan de desarrollo académico (2012-2017). San José, Costa Rica: UNED.

Universidad Estatal a Distancia. (2015). Lineamientos de política institucional (20152019). San José, Costa Rica: UNED.

Evaluar para Aprender: estado actual de catorce asignaturas en la Universidad Estatal a Distancia de Costa Rica Ana Cristina Umaña-Mata, Xinia Calvo-Cruz,

Natalia Salas-Quirós

DOI : http://dx.doi.org/10.22458/caes.v8i2.1809

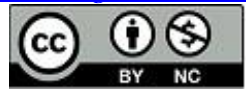

